\title{
Ultrasound-Guided Attenuation Parameter (UGAP) for the quantification of liver steatosis using the Controlled Attenuation Parameter (CAP) as the reference method
}

\author{
Felix Bende, Ioan Sporea, Roxana Sirli, Victor Baldea, Alin Lazar, Raluca Lupușoru, Renata \\ Fofiu, Alina Popescu
}

Department of Gastroenterology and Hepatology, "Victor Babeș” University of Medicine and Pharmacy, Timișoara, Romania

\begin{abstract}
Aim: Nonalcoholic Fatty Liver Disease (NAFLD) is increasing in frequency in daily practice and evaluation of liver steatosis, fibrosis and inflammation severity are essential for prognosis assessment. The aim was to evaluate the usefulness of a new liver steatosis quantification system - Ultrasound-Guided Attenuation Parameter (UGAP) from General Electric Healthcare, using Controlled Attenuation Parameter (CAP) as the reference method. Material and method: 179 consecutive subjects, in whom liver steatosis was assessed in the same session using UGAP, implemented on LOGIQ E10 system (GE Healthcare), and CAP (FibroScan, EchoSens). To discriminate between steatosis stages by CAP, we used the cut-offs recommended by the manufacturer: S1 (mild) - $230 \mathrm{~dB} / \mathrm{m}, \mathrm{S} 2$ (moderate) - $275 \mathrm{~dB} / \mathrm{m}, \mathrm{S} 3$ (severe) - $300 \mathrm{~dB} / \mathrm{m}$. Results: We classified our cohort by means of CAP into the following groups: S0 (no steatosis): 48/176 (27.2\%), S1 (mild): 56/176 (31.6\%), S2 (moderate): $14 / 176$ (7.3\%) and S3 (severe): 59/176 (33.9\%). The mean UGAP values increased with the steatosis grade and for each group were the following: S0: $198.3 \pm 25.7 \mathrm{~dB} / \mathrm{m}, \mathrm{S} 1: 216.86 \pm 26.3 \mathrm{~dB} / \mathrm{m}, \mathrm{S} 2: 237.79 \pm 26.3 \mathrm{~dB} / \mathrm{m}$, and S3: $270.8 \pm 31.62$ $\mathrm{dB} / \mathrm{m}$ respectively $(\mathrm{p}<0.001)$. A very good positive correlation was found between UGAP and CAP values $(\mathrm{r}=0.73, \mathrm{p}<0.0001)$. The best cut-off values for predicting different grades of liver steatosis using CAP as the reference were: $\mathrm{S} 1-192.5 \mathrm{~dB} / \mathrm{m}$ (AUC 0.83); S2 - $231 \mathrm{~dB} / \mathrm{m}$ (AUC 0.90) and S3 - $248 \mathrm{~dB} / \mathrm{m}$ (AUC 0.91). Conclusion: UGAP seems to be a good method for liver steatosis quantification and correlates strongly with CAP values.

Keywords: Ultrasound-guided attenuation parameter (UGAP); liver steatosis quantification; Controlled Attenuation Parameter (CAP); NAFLD.
\end{abstract}

\section{Introduction}

The prevalence of liver steatosis has rapidly increased worldwide; currently nonalcoholic fatty liver disease (NAFLD) is the most prevalent chronic liver disease worldwide, with an estimated global prevalence of 1 billion [1]. NAFLD encompasses a wide histological variety with a spectrum that can go from non-evolutive simple steatosis to progressive non-alcoholic steatohepa-

Received 24.06.2020 Accepted 16.09.2020

Med Ultrason

2021, Vol. 23, No 1, 7-14

Corresponding author: Ioan Sporea

13 Snagov, 300481 Timisoara, Romania

Phone number: +40755353852

E-mail: isporea@umft.ro titis (NASH), which may progress to liver cirrhosis, hepatic failure and hepatocellular carcinoma (HCC) [2].

NAFLD is strongly associated with metabolic syndrome, including type 2 diabetes mellitus (T2DM), dyslipidemia, and obesity. Lifestyles have become increasingly sedentary, and dietary patterns have changed over the past few decades, which has led to an increased prevalence of obesity and insulin resistance in the general population [3], while the number of people with T2DM has quadrupled in the past three decades, diabetes mellitus being the ninth major cause of death [4]. NAFLD has rapidly become the most frequent cause of abnormal liver biochemistry findings in many developed and developing countries [1] and, in the United States, NASH is predicted to become the main indication for liver transplantation [5]. 
For liver steatosis quantification and the differentiation of simple steatosis from NASH, liver biopsy (LB) is the recommended method [6], but considering the large number of cases, it is not a feasible diagnostic tool in such a large population. In addition, because of its invasiveness, LB is not an appropriate, repeatable method that can be used for follow-up.

Therefore, steatosis detection and quantification using precise, repeatable and noninvasive diagnostic tools are mandatory in NAFLD patients. Moreover, it has been demonstrated that liver steatosis in patients with hepatitis $\mathrm{C}$ can lead to more advanced liver fibrosis and a more severe outcome [7].

Imaging techniques are the best and most convenient noninvasive means for liver steatosis evaluation. Conventional B-mode ultrasound was the first used in clinical practice, with $60-94 \%$ sensitivity and $88-95 \%$ specificity in detecting liver steatosis [8], but its accuracy decreases significantly in the case of mild steatosis [9]. Moreover, a skilled examiner is needed for a correct estimation of the steatosis severity, which is only subjective.

Ultrasound attenuation for the quantification of liver steatosis has been developed and studied in the past years. The Controlled Attenuation Parameter (CAP) is a relatively new technique, implemented on the FibroScan device (Echosens, Paris, France), that enables steatosis quantification by measuring ultrasound beam attenuation throughout the liver and has shown a good correlation with histologic grades in adults [10-12]. Moreover, guidelines recommend CAP as an accurate alternative to abdominal ultrasonography for the detection of liver steatosis $[13,14]$.Thus, in the latest WFUMB guidelines on ultrasound elastography, CAP has been recommended as a point-of-care, standardized and reproducible technique for the detection of liver steatosis [15], although its accuracy may be affected by variations in cut-off values of different steatosis grades and different covariates [16]. While CAP does not have the B-mode guidance for choosing the area of liver steatosis measurement, in the last few years ultrasound system manufacturers have developed technologies to quantify the ultrasound beam attenuation incorporated into standard ultrasound machines [17-19]. The advantage of such ultrasound systems is that they can perform steatosis quantification during a standard Bmode abdominal ultrasound examination when the liver seems to be steatosic ("bright liver" with posterior attenuation and an increased hepato-renal index). It is an objective estimation of liver steatosis severity, which can be repeated during follow-up in order to see treatment results.

Ultrasound-guided attenuation parameter (UGAP) measures the attenuation coefficient based on a reference phantom that includes glass bead particles of attenuat- ing materials with known attenuation coefficient. In the UGAP mode, the transmission and reception conditions are fixed to the same values used on the reference phantom, and the acquired echo profiles of the liver are compensated by the reference data. As a result, the compensated sound profiles represent only decay caused by attenuation. If the compensated sound profile is flat, the attenuation is the same as the reference phantom. UGAP includes an automated measurement algorithm to find and analyze the optimum measurement range. The diaphragm is also automatically excluded and the slope is measured across this optimum range to provide a representative attenuation coefficient.

In this study, we aimed to evaluate the usefulness of a new liver steatosis quantification system - UltrasoundGuided Attenuation Parameter (UGAP) from General Electric Healthcare, using the Controlled Attenuation Parameter (CAP) as the reference method.

\section{Material and methods}

\section{Subjects}

A prospective study was conducted between June 2019 and October 2019 in a tertiary Department of Gastroenterology and Hepatology. The study population comprised of 179 consecutive subjects with or without chronic liver disease (mostly NAFLD), who had undergone in the same session UGAP, CAP, Transient Elastography (TE)for liver fibrosis and liver steatosis assessment.

Inclusion criteria for all subjects were the ability to provide informed consent, age $\geq 18$ years old. Inclusion criteria for healthy liver subjects were: no history of liver disease, a normal abdominal ultrasound examination, previously tested negative for hepatitis $\mathrm{B} / \mathrm{C}$ virus and $\mathrm{LS}$ values by $\mathrm{TE}<6.5 \mathrm{kPa}$ [6]. The diagnosis of NAFLD was based on the latest guidelines established by the American Association for the Study of Liver Diseases [14], as follows: fatty changes of the liver observed by imaging; no heavy alcohol consumption (ethanol intake $<210$ g per week for men and $<140 \mathrm{~g}$ per week for women); no other factors inducing fatty changes of the liver such as medications; and no chronic liver disease with clear etiology (hepatitis B virus, hepatitis $\mathrm{C}$ virus, primary biliary cholangitis, primary sclerosing cholangitis or autoimmune hepatitis).

Exclusion criteria were: undergoing antiviral treatment, patients with ascites, patients with signs of biliary obstruction and liver congestion secondary to heart failure and patients with focal liver lesions.

Three operators with good experience in abdominal ultrasound (US), liver fibrosis and steatosis quantifica- 
tion (operator 1: 9 years of experience, operator 2: 5 years of experience, operator 3: 4 years of experience), performed all investigations in the following order: abdominal ultrasound, UGAP measurements followed by TE and CAP measurements so that UGAP measurements could not have been influenced.

All subjects signed informed consent. The study was approved by the Ethics Committee and by the institutional review board (32/16.05.2019) and was performed according to the World Medical Association Declaration of Helsinki.

\section{UGAP measurements}

UGAP measurements were performed using a LOGIQ E10 ultrasound machine (GE Healthcare, Wauwatosa, WI, USA), using a C1-6-D convex array probe. All measurements were performed in fasting conditions for more than 4 hours, on patients in a supine position, with the right arm in maximum abduction, by intercostal approach, in the right liver lobe. A large colored-coded attenuation map, automatically adjusted by the system, was positioned in the right liver lobe, in a homogenous area of the liver, free of large vessels (fig1). Using the quality map option, the best image was selected in order to acquire the attenuation coefficient measurement. Ten measurements were performed using one or two selected images of the liver and the values were automatically stored in the system. Reliable UGAP measurements were defined as the median value of 10 measurements performed in a homogeneous area of liver parenchyma, with an IQR/M $<0.30$. UGAP values are expressed in $\mathrm{dB} / \mathrm{cm} /$ $\mathrm{MHz}$ or in $\mathrm{dB} / \mathrm{m}$.

LOGIQ E10 ultrasound machine can also perform liver stiffness measurements for fibrosis evaluation using an accurate 2D-Shear Wave Elastography (2D-SWE) technique [20-23], but this type of evaluation was not included in the present study.

\section{$T E$ and CAP measurements}

TE and CAP measurements were performed in fasting conditions for more than 4 hours, on patients in supine position, with the right arm in maximum abduction, by intercostal approach, in the right liver lobe. In each patient, we aimed for 10 valid LS measurements, using the $\mathrm{M}$ probe (standard probe - transducer frequency 3.5 $\mathrm{MHz}$ ) or the XL probe (transducer frequency $2.5 \mathrm{MHz}$ ). $\mathrm{M}$ and XL probes were chosen according to the EFSUMB recommendation on $\mathrm{M}$ and XL probe selection [24]. The median value of 10 valid LS measurements was calculated and the results were expressed in kiloPascals $(\mathrm{kPa})$. Reliable measurements were defined as the median value of 10 valid LS measurements, with an interquartile range interval/median ratio $(\mathrm{IQR} / \mathrm{M})<30 \%$ [24]. To discriminate between fibrosis stages, we used the following TE

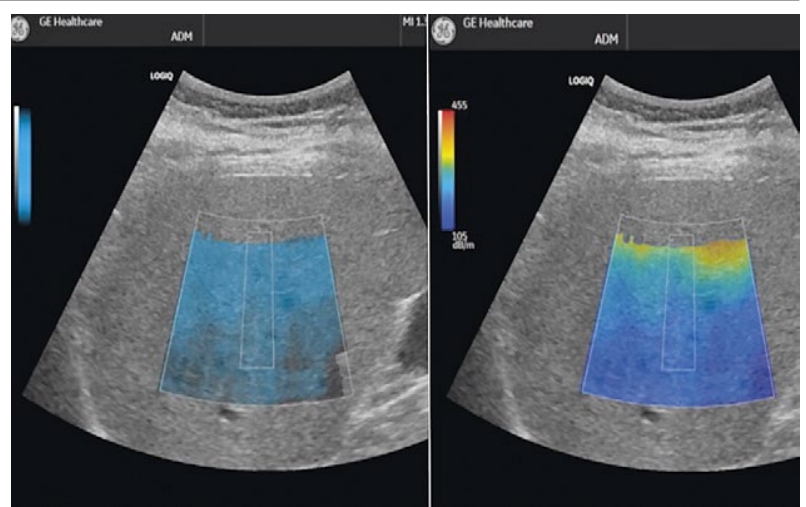

Fig 1. UGAP measurement using the Attenuation map (left) and the quality map (right).

cut-off values [25]: significant fibrosis $(\mathrm{F} \geq 2)-7 \mathrm{kPa}$, severe fibrosis $(\mathrm{F} \geq 3)-9 \mathrm{kPa}$ and cirrhosis $(\mathrm{F}=4)-11.8$ $\mathrm{kPa}$. To discriminate between steatosis stages by CAP we used the cut-offs recommended by the manufacturer: $\mathrm{S} 1$ (mild) - 230dB/m, S2 (moderate) - 275dB/m, S3 (severe) $-300 \mathrm{~dB} / \mathrm{m}$.

\section{Statistical analyses}

The statistical analysis was performed using MedCalc Software, version 12.5.0.0 (MedCalc program, Belgium), SPSS, Version 17.0 (IBM Statistics) and R software packages (v.3.3). Collected data were presented as mean $( \pm \mathrm{SD})$ for continuous variables with a normal distribution, median (IQR) for continuous variables without normal distribution, or absolute frequency (percentage) for nominal variables. The normality of continuous variable distributions was tested using the Kolmogorov-Smirnov's test. The significance of the difference between groups was assessed by using the Student's ttest (means, normal populations), Mann-Whitney U test (medians, non-normal populations), and Pearson's chisquared or Fisher's exact test (proportions). The correlation between two variables was assessed with Pearson's $\mathrm{r}$ correlation coefficient. For method performance and thresholds, receiver operating curve (ROC) analysis was used. We considered a p-value of 0.05 as the threshold for statistical significance and a confidence level of $95 \%$ for estimating intervals.

\section{Results}

A total of 179 consecutive patients were screened during the study period. Of these patients, 2 were excluded due to unreliable CAP and UGAP results, so that 177 patients were included in the final analyses (fig 2). The success rates for CAP and UGAP were similar, $98.8 \%$ (177/179). The reason for failure for CAP and UGAP was BMI $>40 \mathrm{~kg} / \mathrm{m}^{2}$ in both patients. The main charac- 


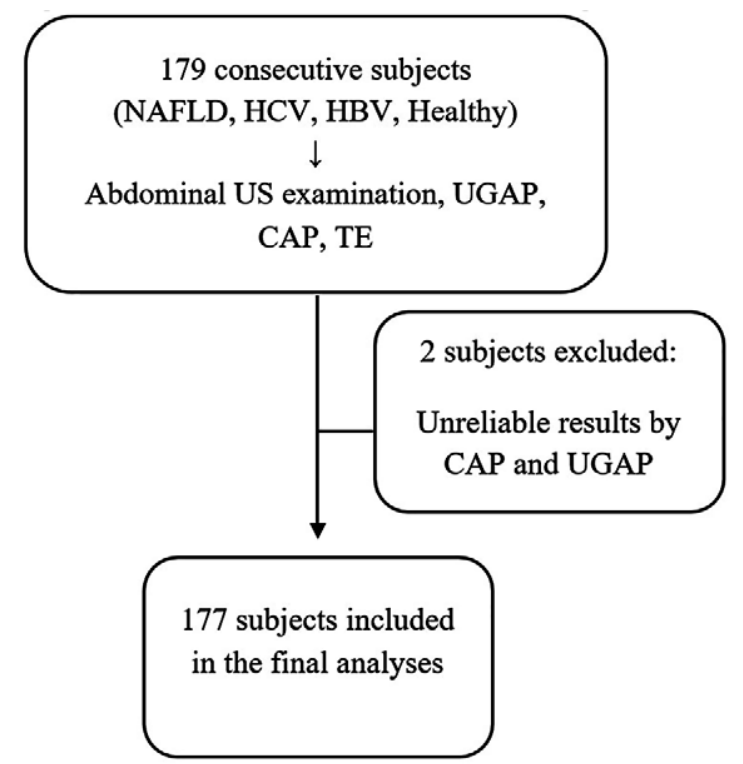

Fig 2. Flow diagram of the study population. NAFLD = nonalcoholic fatty liver disease; $\mathrm{HCV}=$ hepatitis $\mathrm{C}$ virus; $\mathrm{HBV}=$ hepatitis B virus; US = ultrasound; UGAP = ultrasound-guided attenuation parameter; $\mathrm{CAP}=$ control attenuation parameter; $\mathrm{TE}=$ transient elastography.

Table I. Main characteristics of the study population

\begin{tabular}{ll}
\hline Parameter & Value \\
\hline $\mathrm{n}$ & 177 \\
mean age (years) & $52.5 \pm 17.3(20-88)$ \\
gender (male/female) & $88 / 89$ \\
mean BMI $\left(\mathrm{kg} / \mathrm{m}^{2}\right)$ & $28.2 \pm 5.5(17.1-47.6)$ \\
BMI $<25 \mathrm{~kg} / \mathrm{m}^{2}$ & $25.4 \%(45 / 177)$ \\
BMI $25-30 \mathrm{~kg} / \mathrm{m}^{2}$ & $35 \%(62 / 177)$ \\
BMI $\geq 30 \mathrm{~kg} / \mathrm{m}^{2}$ & $39.6 \%(70 / 177)$ \\
Liver disease etiology & \\
Healthy liver subjects & $20.3 \%(36 / 177)$ \\
NAFLD & $48.5 \%(86 / 177)$ \\
HCV & $14.6 \%(26 / 177)$ \\
HBV & $10.1 \%(18 / 177)$ \\
ALD & $6.2 \%(11 / 177)$ \\
Steatosis stage using CAP & \\
S0 & $27.1 \%(48 / 177)$ \\
S1 & $31.6 \%(56 / 177)$ \\
S2 & $8 \%(14 / 177)$ \\
S3 & $33.3 \%(59 / 177)$ \\
Fibrosis stage using TE & \\
F0-F1 & $68.3 \%(121 / 177)$ \\
F2 & $9.6 \%(17 / 177)$ \\
F3 & $8 \%(14 / 177)$ \\
F4 & $14.1 \%(25 / 177)$ \\
\hline
\end{tabular}

Numerical variables with normal distribution are presented as mean value \pm standard deviation, while variables with non-normal distribution are presented as median values and range intervals. $n$ $=$ number; $\mathrm{BMI}=$ body mass index; $\mathrm{NAFLD}=$ non-alcoholic fatty liver disease; $\mathrm{HCV}=$ hepatitis $\mathrm{C}$ virus; $\mathrm{HBV}=$ hepatitis $\mathrm{B}$ virus; $\mathrm{ALD}=$ alcoholic liver disease $\mathrm{CAP}=$ controlled attenuation parameter; $\mathrm{TE}=$ transient elastography. teristics of the study population are presented in Table I. We classified our cohort by means of CAP into 4 groups according to the liver steatosis severity.

The mean UGAP values in our study cohort were significantly lower than the mean CAP values: $231.5 \pm 40.9$ $\mathrm{dB} / \mathrm{m}$ vs. 268.6 $\pm 61.7 \mathrm{~dB} / \mathrm{m}, \mathrm{p}<0.001$. Considering CAP as the reference for liver steatosis quantification, the mean UGAP values increased according to steatosis severity (fig 3 ).

A good positive correlation was found between UGAP and CAP values ( $r=0.73, p<0.0001)$ (fig 4).

The best UGAP cut-off values for predicting different grades of liver steatosis, using $\mathrm{CAP}$ as the reference were: $\mathrm{S} 1$ - $192.5 \mathrm{~dB} / \mathrm{m} ; \mathrm{S} 2-231 \mathrm{~dB} / \mathrm{m} ; \mathrm{S} 3-248 \mathrm{~dB} / \mathrm{m}$ (Table II, fig 5).

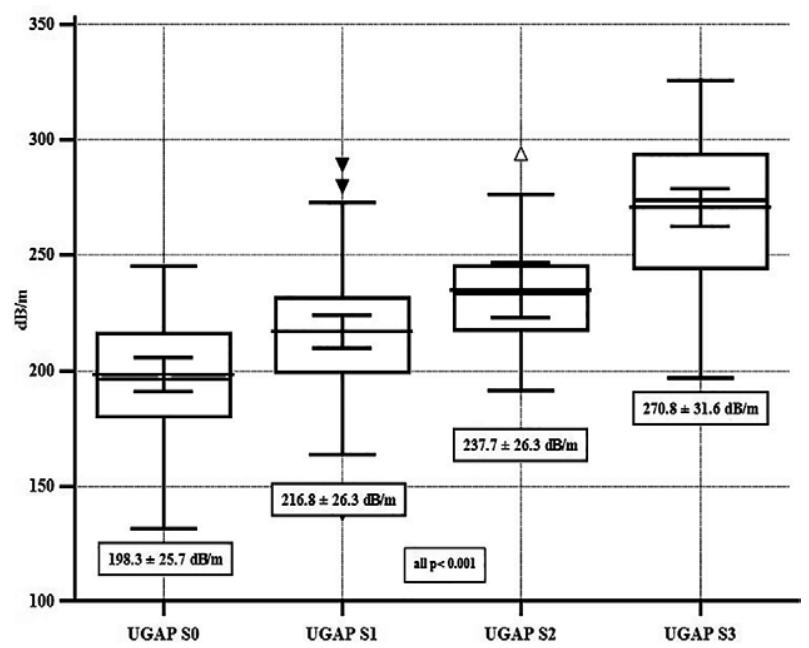

Fig 3. Box plots representing the mean values of UGAP measurements for different stages of liver steatosis.

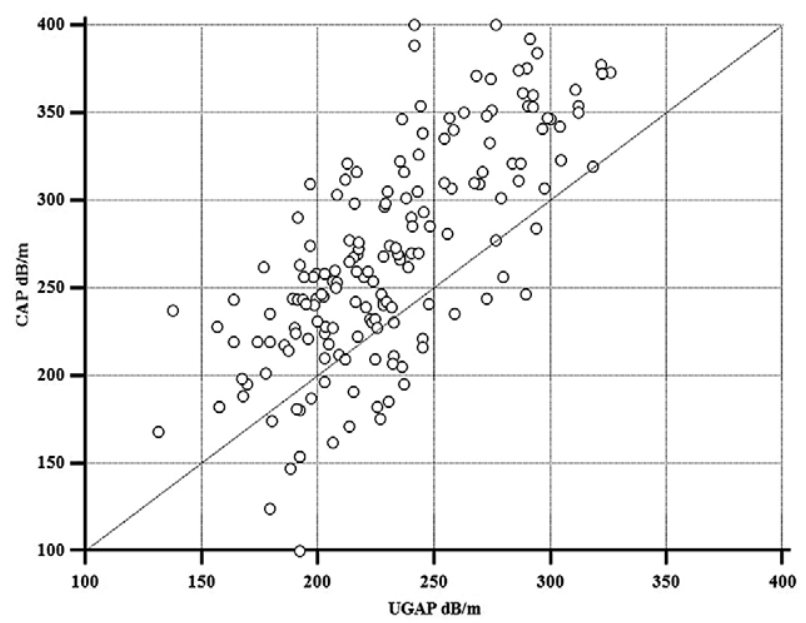

Fig 4. The scatterplot shows the correlation between UGAP and CAP. 

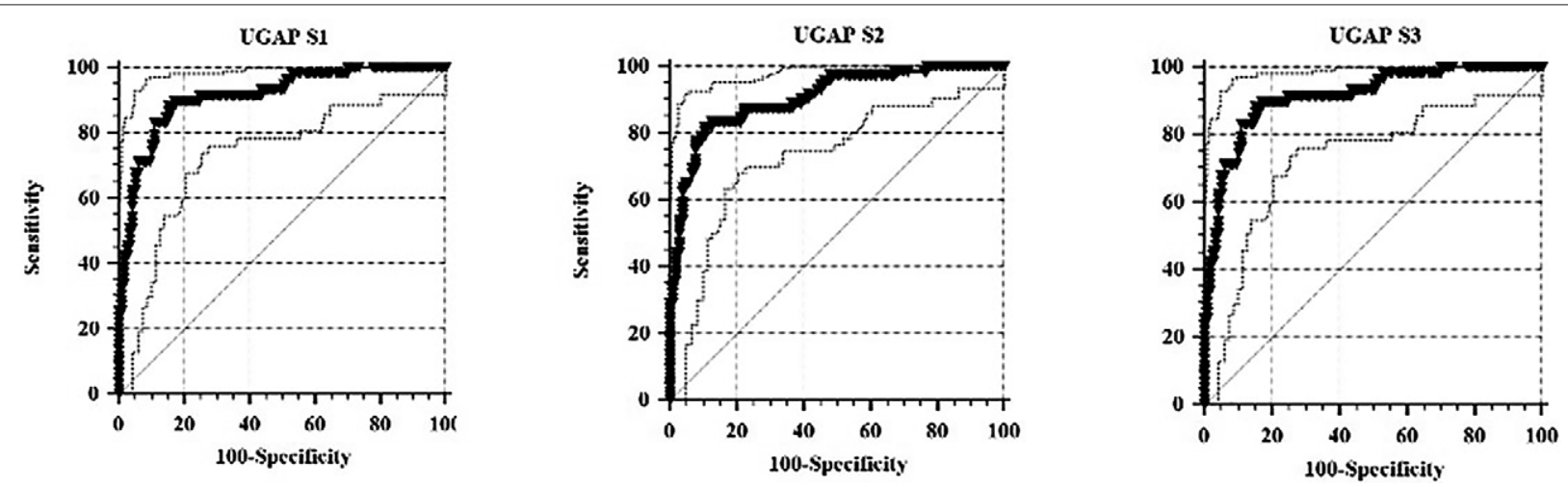

Fig 5. Diagnostic accuracy of UGAP for predicting different grades of liver steatosis.

Table II. UGAP cut-off values for predicting different grades of liver steatosis.

\begin{tabular}{lllllllll}
\hline Steatosis stage & Cut-off (dB/m) & Cut-off $\mathbf{( d B / c m / M H z )}$ & AUC & Se (\%) & Sp (\%) & PPV (\%) & NPV (\%) & p value \\
\hline $\mathrm{S} \geq 1$ & 192.5 & 0.55 & 0.83 & 93.7 & 47.9 & 82.8 & 74.2 & $<0.0001$ \\
$\mathrm{~S} \geq 2$ & 231 & 0.66 & 0.90 & 83.3 & 83.7 & 75.0 & 87.5 & $<0.0001$ \\
$\mathrm{~S}=3$ & 248 & 0.70 & 0.91 & 71.1 & 94.2 & 85.7 & 86.8 & $<0.0001$ \\
\hline
\end{tabular}

$\mathrm{AUC}=$ area under the curve; $\mathrm{Se}=$ sensitivity; $\mathrm{Sp}=$ specificity; $\mathrm{PPV}=$ positive predictive value; $\mathrm{NPV}=$ negative predictive value.

\section{Discussions}

NAFLD has become an emerging and challenging medical problem nowadays due to its increased prevalence, becoming a subject of extensive research in the last years. The need for screening and evaluation of these patients is very high. The main issues regarding populational screening in NASH are to decide how to perform the screening and which population to screen-the general population or the population at risk. It is quite impossible to screen the general population and, maybe, for the moment, we must focus on the population at risk to develop NAFLD, which includes patients with type 2 diabetes mellitus, obese patients, individuals with a metabolic syndrome [26-28]. The screening of this population at risk should be performed, assuredly, by means of noninvasive methods, among which ultrasound-based ones have become very popular in the last 10 years.

Transient Elastography (TE) is a shear wave elastographic technique that allows rapid evaluation of liver fibrosis [24]. CAP was subsequently developed, first implemented on the M probe and later on the XL probe, in order to simultaneously assess liver steatosis [16,29]. In the latter years, CAP has been thoroughly studied and demonstrated a high accuracy for liver steatosis quantification, in comparison with liver biopsy [30,31], subsequently becoming a reference method. However, some problems have been raised regarding CAP, mainly regarding the proposed cut-off values for different groups of patients and regarding the need to use quality criteria $[32,33]$.
On the other hand, FibroScan is a machine used only for fibrosis and steatosis quantification and it is expensive. As an alternative, besides imaging of the abdominal organs (size, structure, focal lesions), modern new ultrasound systems can evaluate liver fibrosis (using point Shear wave elastography or 2D Shear wave elastography) $[20,22,34,35]$ and more recently, can quantify liver steatosis [17-19]. Considering all these capabilities, and also including Contrast-Enhanced Ultrasound, newer ultrasound systems have become really multiparametric ultrasound (MPUS) tools [36].

In this current study, the feasibility of UGAP was very high (98.8\%) and similar to CAP. Unreliable results by means of UGAP were observed in only two patients. The reason for failure in both patients was BMI $>40 \mathrm{~kg} /$ $\mathrm{m}^{2}$. In a previously published study, Fujiwara et. al reported an excellent feasibility of UGAP for liver steatosis quantification $(100 \%)$ and demonstrated a negative association between BMI and UGAP [18]. In our study, almost $40 \%$ of the subjects included were obese, thus demonstrating a very good feasibility of UGAP for liver steatosis assessment in obese patients.

In our study the correlation between CAP measurements and UGAP was 0.73 , corresponding to a strong correlation. Moreover, the UGAP values increased with the steatosis severity. The AUROC of UGAP for predicting grade 3 liver steatosis was higher than 0.90 , corresponding to high diagnostic accuracy. Furthermore, the AUROCs for predicting grade 2 and grade 1 liver steatosis were 0.90 and 0.83 , demonstrating that UGAP has 
a very good diagnostic ability to detect and discriminate among different grades of liver steatosis.

Previously published studies also showed a very high diagnostic accuracy of UGAP for liver steatosis quantification. In a study that included 182 subjects with NAFLD and HCV, using liver biopsy as the reference, the AUROCs of UGAP for diagnosing liver steatosis grade 1,2 and 3 were $0.90,0.95$ and 0.95 respectively [18]. Another published study that enrolled 126 subjects with chronic liver disease, in which magnetic resonance imaging proton density fat fraction (MRI-PDFF) was used as the reference method, UGAP demonstrated AUROCs of $0.92,0.87$ and 0.92 for diagnosing liver steatosis grade 1, 2 and 3 , respectively [37].

Finally, we calculated the best UGAP cut-off values for predicting different stages of liver steatosis, using $\mathrm{CAP}$ as the reference method. The cut-off values for predicting liver steatosis grade 1,2 and 3 were $192.5 \mathrm{~dB} / \mathrm{m}$ $(0.55 \mathrm{~dB} / \mathrm{cm} / \mathrm{MHz}), 231 \mathrm{~dB} / \mathrm{m}(0.66 \mathrm{~dB} / \mathrm{cm} / \mathrm{MHz})$ and $248 \mathrm{~dB} / \mathrm{m}(0.7 \mathrm{~dB} / \mathrm{cm} / \mathrm{MHz})$. Comparing the results in $\mathrm{dB} / \mathrm{cm} / \mathrm{MHz}$ with previously published studies [18,37], the cut-off values seem quite similar. Although the US machine can express and record the US attenuation either in $\mathrm{dB} / \mathrm{m}$ or $\mathrm{dB} / \mathrm{cm} / \mathrm{MHz}$, we recommend for future publications the usage of $\mathrm{dB} / \mathrm{m}$ in order to have more homogenous results and easily compare them with other methods (CAP).

UGAP is an emerging technique that has been recently developed for liver steatosis quantification. The scientific background supporting its usefulness is still weak, with only a few studies published so far, but with promising results. New elastographic and attenuation quantification techniques are rapidly developing and the pressure to release them on the market is increasing. Using liver biopsy in order to validate these methods can be difficult to achieve, taking into account the concerns and the lack of compliance of the patients towards its invasiveness and also the large amount of time needed to recruit subjects. Therefore, many published studies used TE as a reference method for liver fibrosis evaluation. Considering that CAP has been also validated for liver steatosis quantification, with a very good accuracy demonstrated in prospective studies and meta-analyses, we think that CAP can be used as a reference method for liver steatosis quantification [16,29-31]. Some studies used MRIPDFF as a new standard for liver steatosis quantification. Despite the fact that this technique is very sensitive and specific, the cost of this evaluation is high. On the other hand, ultrasound-based steatosis quantification, as well as liver stiffness estimation, are seen as point of care methods, that can be used in the examination room, immediately after an ultrasound examination in subjects at risk.
Several limitations are associated with our study. The main limitation is the lack of liver biopsy or MRI-PDFF as the reference method for liver steatosis quantification. As mentioned before, we used CAP as the reference method since it is a validated and a recommended method for liver steatosis quantification in clinical practice. Another limitation of the study is the number of subjects included, which is quite small for the different etiologies. Taking this into account, there is a need for further studies, with larger cohorts of patients in order to validate our results.

In conclusion, UGAP seems to be a good method for liver steatosis quantification and correlates strongly with CAP values. Feasibility is very good and the examination can be performed immediately after a standard ultrasound examination, being connected with the liver stiffness evaluation.

\section{Conflict of interest: none}

\section{Acknowledgements}

The authors would like to acknowledge that this research is part of an Internal Grant of our University (2EXP/2020).

\section{References}

1. Das K, Das K, Mukherjee PS, et al. Nonobese population in a developing country has a high prevalence of nonalcoholic fatty liver and significant liver disease. Hepatology 2010;51:1593-1602.

2. Perumpail BJ, Khan MA, Yoo ER, Cholankeril G, Kim D, Ahmed A. Clinical epidemiology and disease burden of nonalcoholic fatty liver disease. World J Gastroenterol 2017;23:8263-8276.

3. Anstee QM, Targher G, Day CP. Progression of NAFLD to diabetes mellitus, cardiovascular disease or cirrhosis. Nat Rev Gastroenterol Hepatol 2013;10:330-344.

4. Zheng Y, Ley S, Hu FB. Global aetiology and epidemiology of type 2 diabetes mellitus and its complications. Nat Rev Endocrinol 2018;14:88-98.

5. Charlton MR, Burns JM, Pedersen RA, Watt KD, Heimbach JK, Dierkhising RA. Frequency and outcomes of liver transplantation for nonalcoholic steatohepatitis in the United States. Gastroenterology 2011;141:1249-1253.

6. European Association for the Study of the Liver (EASL); European Association for the Study of Diabetes (EASD); European Association for the Study of Obesity (EASO). EASL-EASD-EASO Clinical Practice Guidelines for the management of non-alcoholic fatty liver disease. J Hepatol 2016;64:1388-1402.

7. Adinolfi LE, Gambardella M, Andreana A, Tripodi MF, Utili R, Ruggiero G. Steatosis accelerates the progression of liver damage of chronic hepatitis $\mathrm{C}$ patients and cor- 
relates with specific HCV genotype and visceral obesity. Hepatology 2001;33:1358-1364.

8. Joy D, Thava VR, Scott BB. Diagnosis of fatty liver disease: is biopsy necessary? Eur J Gastroenterol Hepatol 2003; 15:539-543.

9. Dasarathy S, Dasarathy J, Khiyami A, Joseph R, Lopez R, McCullough AJ. Validity of real time ultrasound in the diagnosis of hepatic steatosis: a prospective study. J Hepatol 2009;51:1061-1067.

10. Sasso M, Beaugrand M, de Ledinghen V, et al. Controlled attenuation parameter (CAP): a novel VCTETM guided ultrasonic attenuation measurement for the evaluation of hepatic steatosis: preliminary study and validation in a cohort of patients with chronic liver disease from various causes. Ultrasound Med Biol 2010;36:1825-1835.

11. Imajo K, Kessoku T, Honda Y, et al. Magnetic Resonance Imaging More Accurately Classifies Steatosis and Fibrosis in Patients With Nonalcoholic Fatty Liver Disease Than Transient Elastography. Gastroenterology 2016;150:626637.e7.

12. Karlas T, PetroffD, Sasso M, et al. Individual patient data meta-analysis of controlled attenuation parameter (CAP) technology for assessing steatosis. J Hepatol 2017;66:1022-1030.

13. Wong VW, Chan WK, Chitturi S, et al. Asia-Pacific Working Party on Non-alcoholic Fatty Liver Disease guidelines 2017-Part 1: Definition, risk factors and assessment. J Gastroenterol Hepatol 2018;33:70-85.

14. Chalasani N, Younossi Z, Lavine JE, et al. The diagnosis and management of nonalcoholic fatty liver disease: Practice guidance from the American Association for the Study of Liver Diseases. Hepatology 2018;67:328-357.

15. Ferraioli G, Wong VW, Castera L, et al. Liver Ultrasound Elastography: An Update to the World Federation for U1trasound in Medicine and Biology Guidelines and Recommendations. Ultrasound Med Biol 2018;44:2419-2440.

16. de Lédinghen V, Vergniol J, Capdepont M, et al. Controlled attenuation parameter (CAP) for the diagnosis of steatosis: A prospective study of 5323 examinations. J Hepatol 2014;60:1026-1031.

17. Bae JS, Lee DH, Lee JY, et al. Assessment of hepatic steatosis by using attenuation imaging: a quantitative, easy-toperform ultrasound technique. Eur Radiol 2019;29:64996507.

18. Fujiwara Y, Kuroda H, Abe T, et al. The B-Mode ImageGuided Ultrasound Attenuation Parameter Accurately Detects Hepatic Steatosis in Chronic Liver Disease. Ultrasound Med Biol 2018;44:2223-2232.

19. Tamaki N, Koizumi Y, Hirooka M, et al. Novel quantitative assessment system of liver steatosis using a newly developed attenuation measurement method. Hepatol Res 2018;48:821-828.

20. Bende F, Sporea I, Sirli R, et. al. Performance of 2D-SWE. GE for predicting different stages of liver fibrosis, using Transient Elastography as the reference method. Med U1trason 2017;19:143-149.

21. Serra C, Grasso V, Conti F, et al. A New Two-Dimensional Shear Wave Elastography for Noninvasive Assessment of
Liver Fibrosis in Healthy Subjects and in Patients with Chronic Liver Disease. Ultraschall Med 2018;39:432-439.

22. Sporea I, Bende F, Popescu A, Lupusoru R, Fofiu R, Sirli R. Are there different cut-off values for staging liver fibrosis using 2D-SWE implemented on different systems from the same manufacturer? Med Ultrason 2020;22:7-12.

23. Moga TV, Stepan AM, Pienar C, et al. Intra- and InterObserver Reproducibility of a 2-D Shear Wave Elastography Technique and the Impact of Ultrasound Experience in Achieving Reliable Data. Ultrasound Med Biol 2018;44:1627-1637.

24. Dietrich CF, Bamber J, Berzigotti A, et al. EFSUMB Guidelines and Recommendations on the Clinical Use of Liver Ultrasound Elastography, Update 2017 (Long Version). Ultraschall Med 2017;38:e16-e47.

25. Kumar R, Rastogi A, Sharma MK, et al. Liver stiffness measurements in patients with different stages of nonalcoholic fatty liver disease: diagnostic performance and clinicopathological correlation. Dig Dis Sci 2013;58:265-274.

26. Patel P, Hossain F, Horsfall LU, et al. A pragmatic approach identifies a high rate of nonalcoholic fatty liver disease with advanced fibrosis in diabetes clinics and at-risk populations in primary care. Hepatol Commun 2018;2:897-905.

27. Younossi ZM, Tampi RP, Racila A, et al. Economic and clinical burden of nonalcoholic steatohepatitis in patients with type 2 diabetes in the U.S. Diabetes Care 2020;43:283-289.

28. Sporea I, Mare R, Popescu A, et al. Screening for Liver Fibrosis and Steatosis in a Large Cohort of Patients with Type 2 Diabetes Using Vibration Controlled Transient Elastography and Controlled Attenuation Parameter in a SingleCenter Real-Life Experience. J Clin Med 2020;9:1032.

29. de Lédinghen V, Hiriart JB, Vergniol J, Merrouche W, Bedossa P, Paradis V. Controlled attenuation parameter (CAP) with the XL probe of the FibroScan: a comparative study with the M probe and liver biopsy. Dig Dis Sci 2017;62:2569-2577.

30. Lupsor-Platon M, Feier D, Stefanescu H, et al. Diagnostic accuracy of CAP measurement by TE for non-invasive assessment of liver steatosis: A prospective study. J Gastrointestin Liver Dis 2015;24:35-42.

31. Karlas T, Petro D, Sasso M, et al. Individual patient data meta-analysis of controlled attenuation parameter (CAP) technology for assessing steatosis. J Hepatol 2017;66:10221030.

32. Wong VW, Irles M, Wong GL, et al. Unified interpretation of liver stiffness measurement by $\mathrm{M}$ and XL probes in nonalcoholic fatty liver disease. Gut 2019;68:2057-2064.

33. Chan WK, Nik Mustapha NR, Mahadeva S, Wong VW, Cheng JY, Wong GL. Can the same controlled attenuation parameter cut-offs be used for M and XL probes for diagnosing hepatic steatosis? J Gastroenterol Hepatol 2018;33:1787-1794.

34. Sporea I, Mare R, Lupusoru R, et al. Comparative study between four ultrasound Shear Waves Elastographic methods for liverfibrosis assessment. Med Ultrason 2018;20:265-271.

35. Herrmann E, de Lédinghen V, Cassinotto C, et al. Assessment of Biopsy-Proven Liver Fibrosis by Two-Dimensional 
Shear Wave Elastography: An Individual Patient DataBased Meta-Analysis. Hepatology 2018;67:260-272.

36. Popescu A. Multiparametric Ultrasound (MPUS) or "The Many Faces" of Ultrasonography. Med Ultrason 2019;21:369-370.
37. Tada T, Kumada T, Toyoda H, et al. Utility of Attenuation Coefficient Measurement Using an Ultrasound-Guided Attenuation Parameter for Evaluation of Hepatic Steatosis: Comparison With MRI-Determined Proton Density Fat Fraction. AJR Am J Roentgenol 2019;212:332-341. 